\title{
Article \\ Ensemble of Deep Convolutional Learning Classifier System Based on Genetic Algorithm for Database Intrusion Detection
}

\author{
Seok-Jun Bu (1), Han-Bit Kang (1) and Sung-Bae Cho*(1) \\ Department of Computer Science, Yonsei University, Seoul 03722, Korea; sjbuhan@yonsei.ac.kr (S.-J.B.); \\ prixt@yonsei.ac.kr (H.-B.K.) \\ * Correspondence: sbcho@yonsei.ac.kr
}

check for updates

Citation: Bu, S.-J.; Kang, H.-B.; Cho, S.-B. Ensemble of Deep Convolutional Learning Classifier System Based on Genetic Algorithm for Database Intrusion Detection. Electronics 2022, 11, 745. https:// doi.org/10.3390/electronics11050745

Academic Editor: Aryya Gangopadhyay

Received: 8 July 2021

Accepted: 26 February 2022

Published: 28 February 2022

Publisher's Note: MDPI stays neutral with regard to jurisdictional claims in published maps and institutional affiliations.

Copyright: (C) 2022 by the authors. Licensee MDPI, Basel, Switzerland. This article is an open access article distributed under the terms and conditions of the Creative Commons Attribution (CC BY) license (https:// creativecommons.org/licenses/by/ $4.0 /)$.

\begin{abstract}
Methods of applying deep learning to database protection have increased over the years To secure role-based access control (RBAC) by learning the mapping function between query features and roles, it is known that the convolutional neural networks combined with learning classifier systems (LCS) can reach formidable accuracy. However, current methods are focused on using a singular model architecture and fail to fully exploit features that other models are capable of utilizing. Different deep architectures, such as ResNet and Inception, can exploit different spatial correlations within the feature space. In this paper, we propose an ensemble of multiple models with different deep convolutional architectures to improve the overall coverage of features used in role classification. By combining models with heterogeneous topologies, the ensemble-LCS model shows significantly increased performance compared to previous single architecture LCS models and achieves better robustness in the case of training data imbalance.
\end{abstract}

Keywords: ensemble classifier; deep learning; genetic algorithm; learning classifier system; role-based access control; database intrusion detection

\section{Introduction}

Database security is a constantly changing field, with attackers searching and exploiting weak points in current defense measures and defenders that develop new methods to protect the database from newly discovered exploits and trying to fortify security measures to future threats [1]. Various methods have already been developed, ranging from simple methods, such as password protection, creating policies that determine the limits of access to the user, and more recent, advanced methods that try to implement the growing field of deep learning $[2,3]$.

Well-known threats to relational databases can be classified into outsider attacks and insider attacks [4]. Outsider attacks, such as SQL injections, can be relatively easily detected with traditional methods. Insider attacks are malicious actions or transactions that are carried out by actors with legitimate access within the infrastructure [5,6]. Insider attacks are more challenging to model and detect $[7,8]$, as both individual and sequential patterns of the users' queries must be identified [9]. Role-based access control (RBAC) attempts to model user-database access by assigning roles to each user and limiting what each role can access. As such, by identifying which role the user has and classifying what role the user query would require, one can develop an intruder detection system (IDS) by verifying whether the user's role and query's role match, as shown in Figure 1.

Traditionally, the query role classification model was designed using hard-coded query grammar analysis [10], often requiring an understanding of the underlying database and transaction structure to model the characteristics of queries. More sophisticated methods, such as using multiple models for parsing SQL queries, have also been applied [11]. Recently, with the development of neural networks, deep learning methods has been applied to this domain, with promising results. In combination with neural network-based 
classification models, Learning Classifier Systems (LCS) that use a genetic algorithm for feature selection [12] have been used to increase the adaptability of IDS to account for changing usage patterns of both legitimate and illegitimate users.

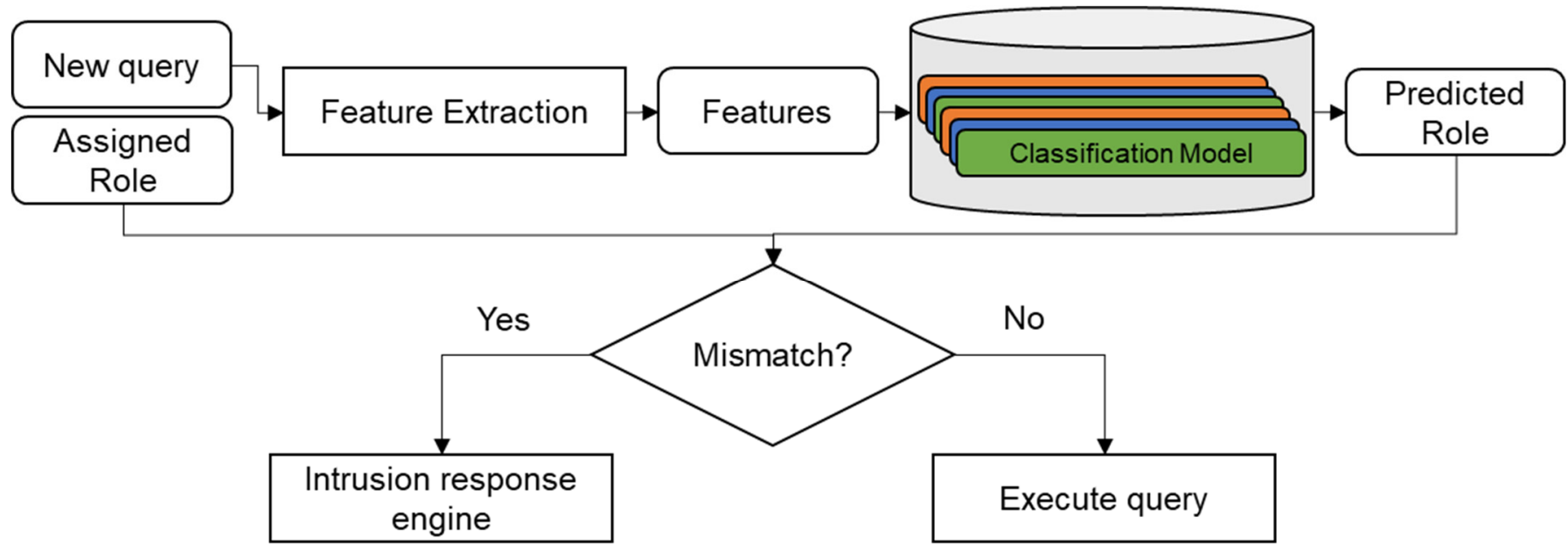

Figure 1. Diagram of an intruder detection system based on role classification.

However, even with such advances, current methods still have limitations; recent models are built on single model architectures and, therefore, cannot provide coverage for all spatial relations within the feature space. In this paper, we propose a method that mitigates this shortcoming by using multiple model architectures in conjunctions, thus increasing the system's feature space coverage. The main contributions of this paper are as follows:

- We utilize models of different architectures and their tendency to associate with TPC-E schema roles categories by collecting heterogeneous models into an ensemble.

- We exploit the effect of model ensembles targeting both learning and adaptability for RBAC-based intrusion detection.

\section{Related Works}

Table 1 summarizes related works on applying machine learning for SQL transaction role classification.

Before queries can be classified, it is necessary to extract salient features from diverse query inputs. Ronao et al. [4] used PCA to analyze various potential queries and managed to extract a set number of features that can be used by other classification models, such as Random Forest Ensembles. Based on these features, other classification models, such as SVM [13], MLP [14], and CNN-LSTM [15], have been used to develop more advanced classifications methods.

CNNs, compared to other methods, showed the most promising accuracy. However, human inputs and data collected in real-world environments are often incomplete or skewed. Query characteristics may also change over time. As such, further optimization of $\mathrm{CNN}$ models is necessary.

Evolutionary algorithms are often utilized to optimize hyperparameters of the model to improve the overall accuracy of the model. Khare et al. [16] used Spider Monkey Optimization to reduce the dimensionality of complex input data, improving the neural network's classification accuracy for the NSL-KDD dataset. Selvakumar et al. [17] used the Firefly algorithm for dimensionality reduction on the KDD CUP 99 dataset with Bayesian Networks for network anomaly detection. 
Table 1. Related works on machine learning for the SQL transaction role classification.

\begin{tabular}{|c|c|c|c|c|}
\hline Approach & $\begin{array}{l}\text { Detection } \\
\text { Mechanism }\end{array}$ & Optimization Method & Objective & Accuracy \\
\hline \multirow{4}{*}{ Machine Learning } & $\begin{array}{c}\text { PCA, } \\
\text { Random Forest [4] }\end{array}$ & \multirow{4}{*}{-} & $\begin{array}{l}\text { Query feature extraction, } \\
\text { role classification tree ensemble }\end{array}$ & 0.7682 \\
\hline & SVM [13] & & $\begin{array}{l}\text { Learn kernel-based query } \\
\text { feature selection boundary }\end{array}$ & 0.7800 \\
\hline & MLP [14] & & $\begin{array}{l}\text { Classification based on } \\
\text { query features }\end{array}$ & 0.8002 \\
\hline & CNN-LSTM [15] & & Learn spatial relationship of query features & 0.9265 \\
\hline \multirow{5}{*}{$\begin{array}{c}\text { CNN } \\
\text { Optimization }\end{array}$} & \multirow{3}{*}{$\mathrm{CNN}$} & ERL [18] & Optimization of learning process & 0.9450 \\
\hline & & LCS [19] & Optimization of feature selection & 0.9255 \\
\hline & & PSO [20] & Optimization of network topology & 0.9400 \\
\hline & CNN Ensemble & GA [21] & Optimization of ensemble rules & 0.9663 \\
\hline & $\begin{array}{c}\text { CNN, } \\
\text { Inception, } \\
\text { ResNet }\end{array}$ & LCS [22] & Compare various GA optimized CNN models & $\begin{array}{l}0.9200 \\
0.9527 \\
0.9427\end{array}$ \\
\hline
\end{tabular}

The combination of the evolutionary algorithm and CNNs can also be found in dataset query role classification research. Choi et al. [18] combined evolutionary reinforcement learning to optimize the learning process of CNNs, attempting to find the ideal learning rate for each training input using the evolutionary process. Bu et al. [19] combined the CNN model with a Learning Classifier System (CN-LCS), allowing the model to learn which input features to focus on to improve model accuracy. Kim et al. [20] used Particle Swarm Optimization to discover improved model topologies. The evolutionary algorithm can also be used in ensemble optimization, such as in the work by Bu et al. [21] to optimize ensembles of CN-LCS.

The CN-LCS method can be expanded for more advanced CNN architectures. Kim et al. [22] expanded the CN-LCS method for classification networks inspired by advanced image classification models, such as Inception Networks [23] (Inception) and Residual Networks [24] (ResNet), observing improved accuracy for models based on more advanced architecture and complexity.

The improvement of model architecture in tandem with additional optimization via evolutionary algorithms showed increased accuracy of the system. However, most research has focused on the optimization of a single model. Even in research where ensembles were used, it used a single type of architecture. We utilized multiple types of CNN model architectures, increasing the diversity of models used by the ensemble. This expands the feature analyzing the capability of the classification system.

\section{The Proposed Method}

The proposed method exploits the different capabilities of diverse $\mathrm{CNN}$ architectures by combining heterogeneous models into an ensemble. It is known that the intrusion detection system accuracy can be enhanced by utilizing the ensemble of multiple models $[25,26]$. The method can be divided into three components: the dataset generation and pre-processing component generates the relevant dataset based on specification and converts the raw input queries into model readable features; model generation uses a genetic algorithm to generate a population of different query feature selections and generate a corresponding population of models; finally, the ensemble collection component uses the results of each generated models to select and combine relevant models into an ensemble with respect to accuracy and diversity. 


\subsection{Dataset Generation and Pre-Processing}

Before generating the models, it is necessary to generate the SQL transaction data that will be used during model training. Synthetic queries are generated that model the roles of the SQL transactions. These queries will mimic and model the transactions that users will have with the database in realistic scenarios while having the advantage of being balanced by class.

The TPC Benchmark E (TPC-E) [27] is an online transaction processing (OLTP) benchmark created by the Transaction Processing Performance Council (TPC). It is designed to evaluate system functionalities in a manner representative of a complex OLTP application environment, such as brokerage firms. The TPC-E schema will be used for database interaction modeling and pseudocode. The schema is based on the activities that can be observed by brokerage firms that handle transactions related to customers, markets, and finances (such as customer account, customer transaction order executions, or market-customer interactions). Table 2 is a summary of the roles within this schema, what specification each role has, and how many queries were generated for the datasets.

Table 2. Roles and specifications for the generated queries based on TPC-E [27].

\begin{tabular}{|c|c|c|c|}
\hline Transactions & Specifications & Transactions & Specifications \\
\hline \multicolumn{2}{|c|}{ Read-only Transactions } & \multicolumn{2}{|c|}{ Read/Write Transactions } \\
\hline \multirow{2}{*}{ Broker-volume (0) } & SELECT only & \multirow{2}{*}{ Trade-order (6) } & SELECT, INSERT \\
\hline & $714 \mathrm{~kb}$ & & $759 \mathrm{~kb}$ \\
\hline \multirow{2}{*}{ Customer-position (1) } & SELECT only & \multirow{2}{*}{ Trade-update (7) } & SELECT, UPDATE \\
\hline & $566 \mathrm{~kb}$ & & $499 \mathrm{~kb}$ \\
\hline \multirow{2}{*}{ Market-watch (2) } & SELECT only & \multirow{2}{*}{ Data-maintenance (8) } & SELECT, UPDATE \\
\hline & $863 \mathrm{~kb}$ & & $246 \mathrm{~kb}$ \\
\hline \multirow[t]{2}{*}{ Security-detail (3) } & SELECT only & \multirow[t]{2}{*}{ Market-feed (9) } & $\begin{array}{l}\text { SELECT, INSERT, } \\
\text { UPDATE, DELETE }\end{array}$ \\
\hline & $571 \mathrm{~kb}$ & & $456 \mathrm{~kb}$ \\
\hline \multirow[t]{2}{*}{ Trade-status (4) } & SELECT only & \multirow[t]{2}{*}{ Trade-result (10) } & $\begin{array}{l}\text { SELECT, INSERT, } \\
\text { UPDATE, DELETE }\end{array}$ \\
\hline & $571 \mathrm{~kb}$ & & $419 \mathrm{~kb}$ \\
\hline \multirow{2}{*}{ Trade-lookup (5) } & SELECT only & \multirow{2}{*}{\multicolumn{2}{|c|}{ Total 11,000 queries generated }} \\
\hline & $490 \mathrm{~kb}$ & & \\
\hline
\end{tabular}

After the dataset generation, the queries are pre-processed into a set number of features. The feature extraction process follows the method proposed Ronao et al. [4], extracting a total of 277 feature values. This method was adopted by previous research by some of the authors, such as those by Bu et al. [7]., showing promising results. This query pre-processing method requires two stages:

First, a tree-wise SQL parsing process reduces the transaction queries into groups based on a defined pattern. Since SQL transactions are written in a structured language, this stage can be implemented with multiple if-then-else statements.

Second, a feature vector containing the following fields are generated: command features (SQL_CMD), projection relation features (PROJ_REL_DEC), projection attribute features (PROJ-ATTR-DEC), selection attribute features (SEL_ATTR_DEC), ORDER BY clause features (ORDBY_ATTR_DEC), GROUP BY clause features (GRPBY_ATTR_DEC), and value counter features (VALUE_CTR).

Figure 2 illustrates the query pre-processing component, and an example of a query and its extracted features is presented in Table 3. 
Through this process, each SQL query can be expressed as a 277-dimensional input feature vector. This vector can then be used by the model generation process to be used for training and evaluation.

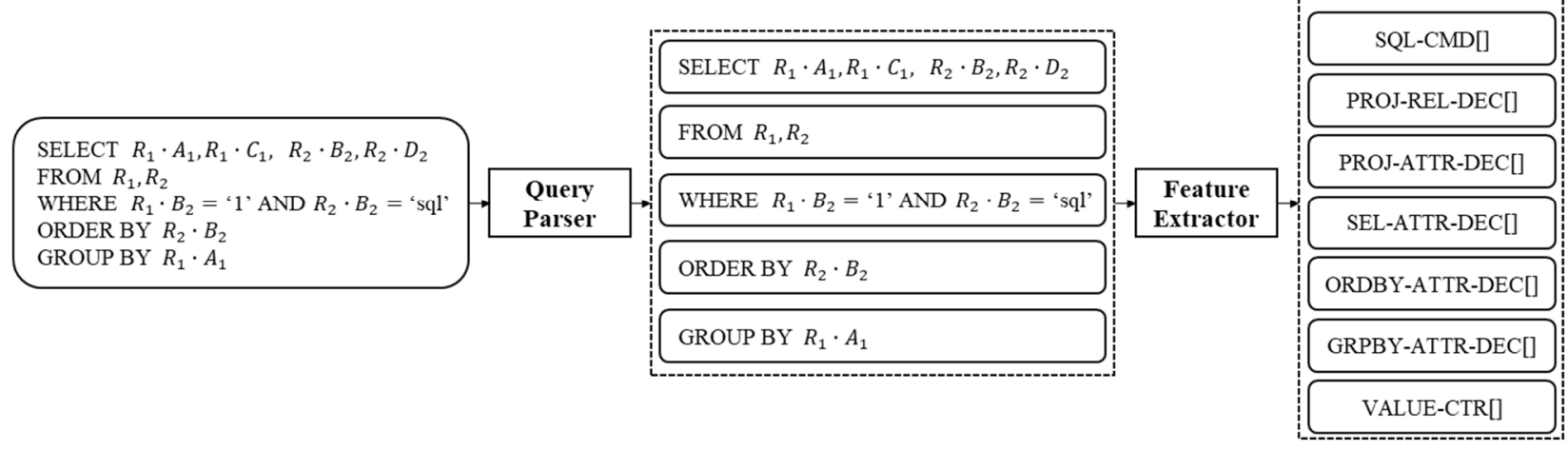

Figure 2. Query pre-processing component.

Table 3. Example query and its extracted features.

\begin{tabular}{|c|c|c|c|}
\hline SQL Query & Feature Fields & Feature Element & Feature Values \\
\hline \multirow{16}{*}{$\begin{array}{c}\text { SELECT } R_{1} \cdot A_{1}, R_{1} \cdot C_{1}, R_{2} \cdot \\
B_{2}, R_{2} \cdot D_{2} \\
\text { FROM } R_{1} \cdot R_{2} \\
\text { WHERE } R_{1} \cdot B_{2}={ }^{\prime}{ }^{\prime} \\
\text { AND } R_{2} \cdot B_{2}=\text { 'sql' } \\
\text { ORDER BY } R_{2} \cdot B_{2} \\
\text { GROUP BY } R_{1} \cdot A_{1}\end{array}$} & \multirow{2}{*}{ SQL-CMD[] } & Query mode & 1 \\
\hline & & $\begin{array}{l}\text { Query length, } \\
Q_{L}\end{array}$ & Character count \\
\hline & \multirow{2}{*}{ PROJ-REL-DEC[] } & $\begin{array}{c}\text { Number of projected relations, } \\
P_{R}\end{array}$ & 2 \\
\hline & & $\begin{array}{l}\text { Position of projected relations, } \\
P_{R I D}\end{array}$ & {$[0011]=3$} \\
\hline & \multirow{3}{*}{ PROJ-ATTR-DEC[] } & $\begin{array}{l}\text { Number of attributes in projection clause, } \\
P_{A}\end{array}$ & 4 \\
\hline & & $\begin{array}{l}\text { Number of projection clause attributes per table, } \\
\qquad P_{A}[]\end{array}$ & {$[2,2]$} \\
\hline & & $\begin{array}{l}\text { Position of projection clause attributes, } \\
P_{A I D}[]\end{array}$ & {$[1010,0101]=[10,5]$} \\
\hline & \multirow{3}{*}{ SEL-ATTR-DEC[] } & $\begin{array}{l}\text { Number of attributes in selection clause, } \\
\qquad S_{A}\end{array}$ & 2 \\
\hline & & $\begin{array}{l}\text { Number of attributes in selection clause per table, } \\
\qquad S_{A}[]\end{array}$ & {$[1,1]$} \\
\hline & & $\begin{array}{l}\text { Position of selection clause attributes, } \\
\qquad S_{A I D}[]\end{array}$ & {$[0100,0100]=[4,4]$} \\
\hline & \multirow{3}{*}{$\begin{array}{l}\text { ORDER-ATTR- } \\
\text { DEC[] }\end{array}$} & $\begin{array}{l}\text { Number of attributes in ORDER BY clause, } \\
\qquad O_{A}\end{array}$ & 1 \\
\hline & & $\begin{array}{l}\text { Number of attributes in ORDER BY clause per table, } \\
\qquad O_{A}[]\end{array}$ & {$[0,1]$} \\
\hline & & $\begin{array}{l}\text { Position of ORDER BY clause, } \\
\qquad O_{A I D}[]\end{array}$ & {$[0000,0100]=[0,4]$} \\
\hline & \multirow{3}{*}{$\begin{array}{l}\text { GRPBY-ATTR- } \\
\text { DEC[] }\end{array}$} & $\begin{array}{l}\text { Number of GROUP BY clause attributes, } \\
\qquad G_{A}\end{array}$ & 1 \\
\hline & & $\begin{array}{l}\text { Number of GROUP BY clause attributes per table, } \\
\qquad G_{A}[]\end{array}$ & {$[1,0]$} \\
\hline & & $\begin{array}{l}\text { Position of GROUP BY clause attributes, } \\
G_{A I D}[]\end{array}$ & {$[1000,0000]=[8,0]$} \\
\hline
\end{tabular}


Table 3. Cont.

\begin{tabular}{|c|c|c|c|}
\hline SQL Query & Feature Fields & Feature Element & Feature Values \\
\hline & \multirow{5}{*}{ VALUE-CTR[] } & $\begin{array}{c}\text { Number of string values, } \\
S_{V}\end{array}$ & 1 \\
\hline & & $\begin{array}{l}\text { Length of string values, } \\
\qquad S_{L}\end{array}$ & 3 \\
\hline & & $\begin{array}{l}\text { Number of numeric values, } \\
\qquad N_{V}\end{array}$ & 1 \\
\hline & & $\begin{array}{c}\text { Number of JOINs, } \\
J\end{array}$ & 0 \\
\hline & & $\begin{array}{c}\text { Number of ANDs and ORs, } \\
\qquad A O\end{array}$ & 1 \\
\hline
\end{tabular}

\subsection{Genetic Algorithm-Based Model Generation}

It is preferable that the generated sets of models use different input features but still maintain high accuracy. To achieve this, a genetic algorithm-based LCS is used. Each of the 277 input features is assigned to a bit in a 277-bit long chromosome. For each chromosome, a CNN model is trained using the selected features as the input and the transaction role as the target output. The accuracy of each model is used as the fitness of their respective chromosome, which is then used for the evolutionary selection process. By repeating this process, the LCS generates a set of input feature selection rules and CNN models paired with each rule. This model generation process is illustrated in Figure 3.

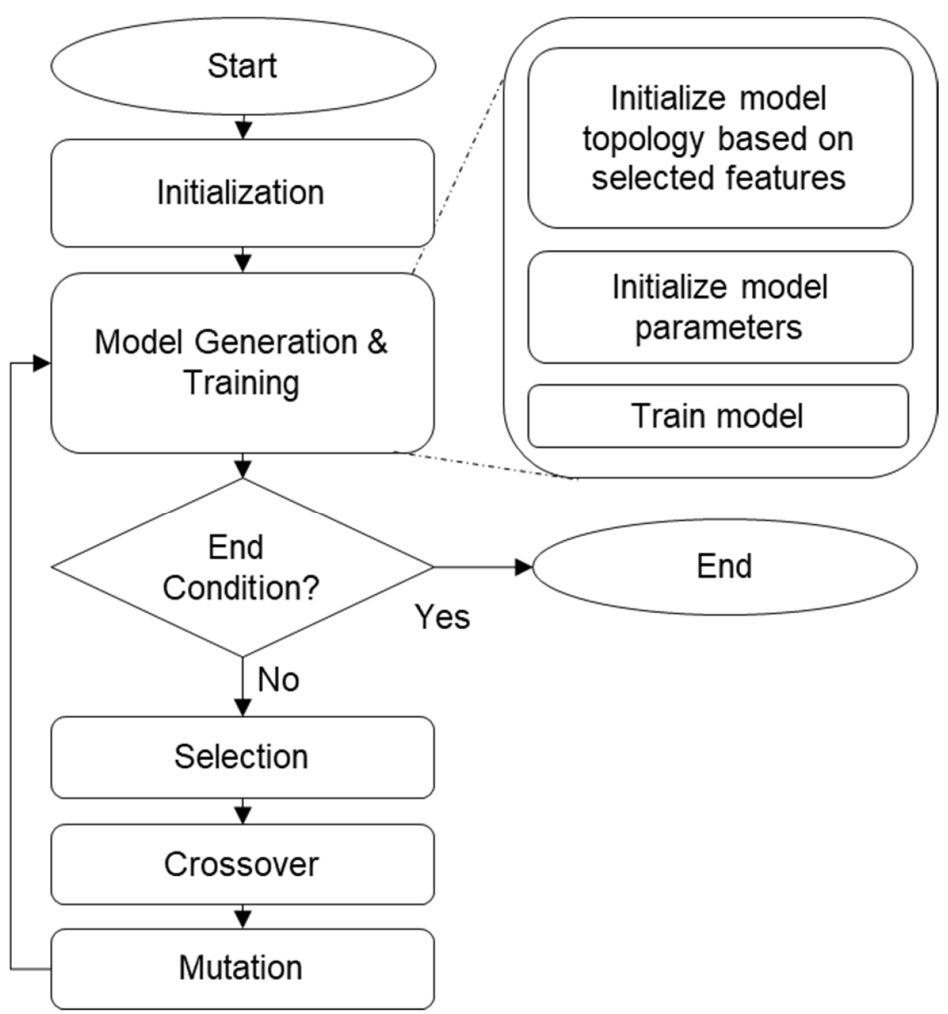

Figure 3. Flowchart of the model generation process.

As a result of using GA for feature selection, multiple models with the same architecture but different parameters are generated. These models are collected into a set of potential models that will be used for the ensemble model. In addition to generating multiple models of the same architecture, models with different CNN architectures are 
trained in separate LCS processes and collected into the same set. This increases the number of potential models and improves the diversity in model types.

The architecture of each CNN used is detailed in Tables 4-7, with the signature blocks of each model illustrated in Figure 4. CNN, Inception, and ResNet-based models are based on the architecture used by Kim et al. [22] The DenseNet classifiers are based on the Dense Network image classification model [28], which connects each layer within the Dense block with multiple residual connections.

Table 4. Summary of the default hyperparameters of the CNN classifier.

\begin{tabular}{ccc}
\hline Type & Configuration & Activation \\
\hline Convolution & Filter $32 \times 1 \times 2$, stride $1 \times 1$ & tanh \\
\hline Convolution & Filter $32 \times 1 \times 2$, stride $1 \times 1$ & tanh \\
\hline Convolution & Filter $32 \times 1 \times 2$, stride $1 \times 1$ & tanh \\
\hline Maxpool & Pooling size $1 \times 2$, stride $1 \times 1$ & - \\
\hline Fully-connected & 256 hidden units & ReLU \\
\hline Fully-connected & 11 hidden units & Softmax \\
\hline
\end{tabular}

Table 5. Summary of the default hyperparameters of the Inception classifier.

\begin{tabular}{ccc}
\hline Type & Configuration & Activation \\
\hline Convolution & Filter $32 \times 1 \times 2$, stride $1 \times 1$ & tanh \\
\hline Maxpool & Pooling size $1 \times 2$, stride $1 \times 1$ & - \\
\hline Convolution & Inception module & \\
\hline Maxpool & Filter $32 \times 1 \times 2$, stride $1 \times 1$ & tanh \\
\hline Fully-connected & Pooling size $1 \times 2$, stride $1 \times 1$ & \\
\hline Fully-connected & Inception module & ReLU \\
\hline
\end{tabular}

Table 6. Summary of the default hyperparameters of the ResNet classifier.

\begin{tabular}{ccc}
\hline Type & Configuration & Activation \\
\hline Convolution & Filter $32 \times 1 \times 2$, stride $1 \times 1$ & tanh \\
\hline & Residual block & \\
\hline Maxpool & Residual block & - \\
\hline Fully-connected & Pooling size $1 \times 2$, stride $1 \times 1$ & ReLU \\
\hline Fully-connected & 256 hidden units & Softmax \\
\hline
\end{tabular}

Table 7. Summary of the default hyperparameters of the DenseNet classifier.

\begin{tabular}{ccc}
\hline & Configuration & Activation \\
\hline Convolution & Filter $32 \times 1 \times 2$, stride $1 \times 1$ & tanh \\
\hline & Dense block & \\
\hline Maxpool & Dense block & - \\
\hline Fully-connected & Pooling size $1 \times 2$, stride $1 \times 1$ & ReLU \\
\hline Fully-connected & 256 hidden units & Softmax \\
\hline
\end{tabular}




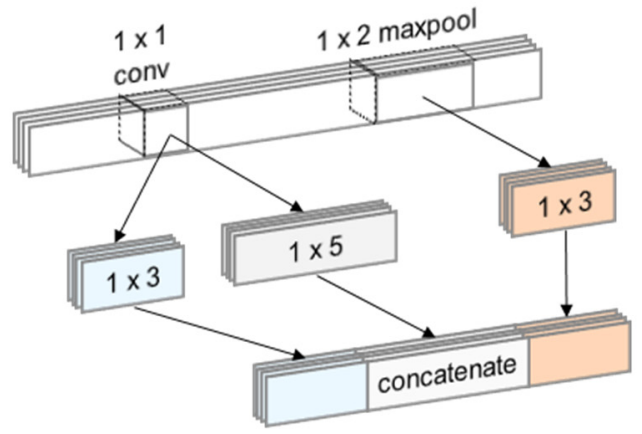

(a)

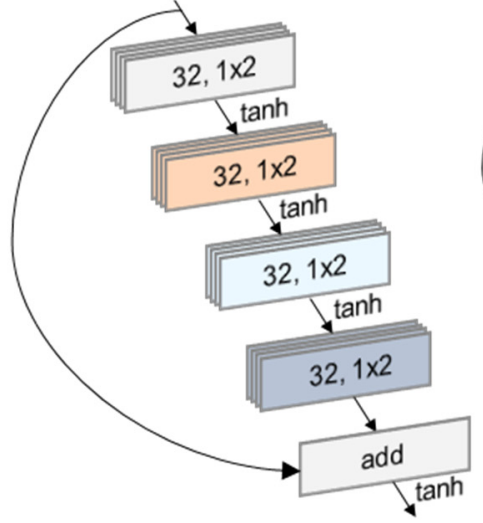

(b)

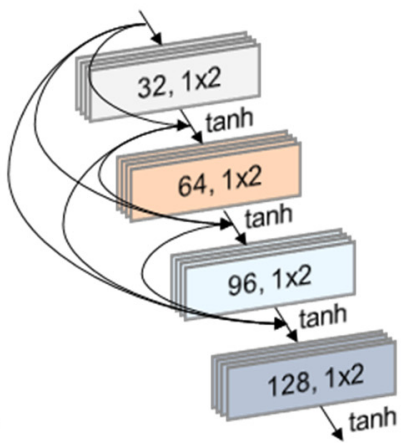

(c)

Figure 4. Signature blocks within the CNN models: (a) Inception block, (b) ResNet block, (c) DenseNet block.

During training, the result of each final model is recorded. This result will be used by the model ensemble component to calculate the diversity between each model. The overall structure of the model generation component is illustrated in Figure 5.

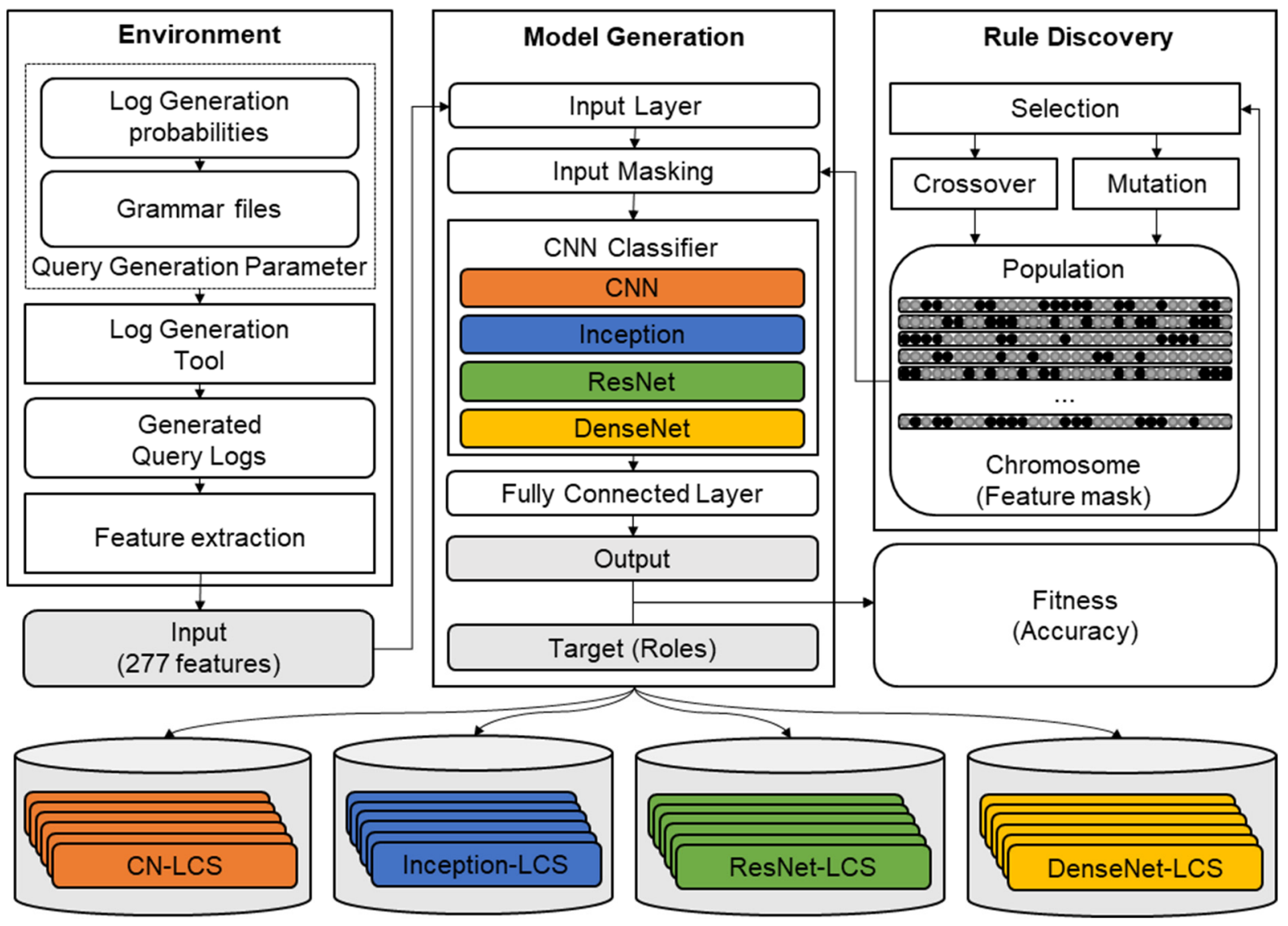

Figure 5. Diagram of the model generation component.

\subsection{Model Ensemble}

Simply collecting all the models into a single ensemble is not enough to utilize the characteristics of each model, as it runs the risk of the minority model being suppressed by the majority. It is preferable that each model in the ensemble is individually accurate, and at the same time, show errors at different input instances, thus displaying diversity 
in results [29]. To enhance such diversity within the ensemble, the average $Q$-statistic $\left(Q_{a v}\right)[30]$ is used. Yule's $Q$-statistic [31] is calculated as denoted in Equation (1), based on the output between each pair of models $\left(D_{i}, D_{k}\right)$ in the candidate set, where $D_{i}$ denotes $i$ th classifier and $N$ denotes the number of observations as denoted in Table 8.

$$
Q_{i, k}=\frac{N^{11} N^{00}-N^{01} N^{10}}{N^{11} N^{00}+N^{01} N^{10}}
$$

Table 8. Relevant number of observations between two models used in $Q$-statistic calculation.

\begin{tabular}{ccc}
\hline & $\boldsymbol{D}_{\boldsymbol{k}}$ Correct (1) & $\boldsymbol{D}_{\boldsymbol{k}}$ Wrong (0) \\
\hline$D_{i}$ correct (1) & $N^{11}$ & $N^{10}$ \\
\hline$D_{i}$ wrong (0) & $N^{01}$ & $N^{00}$ \\
\hline & Total, $N=N^{00}+N^{01}+N^{10}+N^{11}$ \\
\hline
\end{tabular}

The $Q$-statistic measures how correlated the results of the two classifiers are; when the two classifiers correctly predict the roles of the same instances, the $Q$ value approaches 1 ; when the classifiers commit errors at different instances, the $Q$ value approaches -1 . Afterward, starting with the model with the highest accuracy, a set of models are collected such that $Q_{a v}$ of the ensemble is reduced until the number of models within the ensemble is more or equal to the minimum number of required models, and no other models can be added to the set without increasing the ensemble's $Q_{a v}$ :

$$
Q_{a v}=\frac{2}{L(L-1)} \sum_{i=1}^{L-1} \sum_{k=i+1}^{L} Q_{i, k}
$$

The details of the ensemble collection process for selecting the heterogeneous model considering diversity are described in Algorithm 1. When using the ensemble, the results of each model are aggregated using weighted hard voting, with the accuracy of each model used as the weight. Weighted hard voting can be calculated as denoted in Equation (3), where $X$ is the input to the ensemble, $y$ is the resulting role classified by the ensemble, and $p_{i}(y \mid X)$ is the probability distribution of each model. Figure 6 illustrates the overall component structure.

$$
y=\operatorname{argmax}\left(\sum_{i=1}^{L} w_{i} \cdot \operatorname{onehot}\left(\operatorname{argmax}\left(p_{i}(y \mid X)\right)\right)\right)
$$

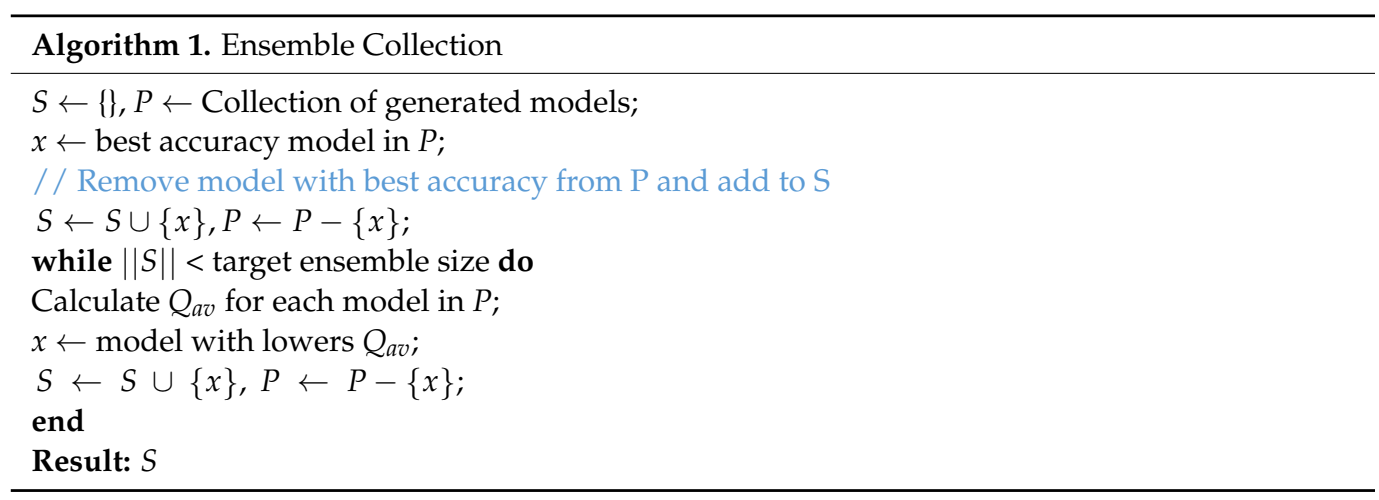




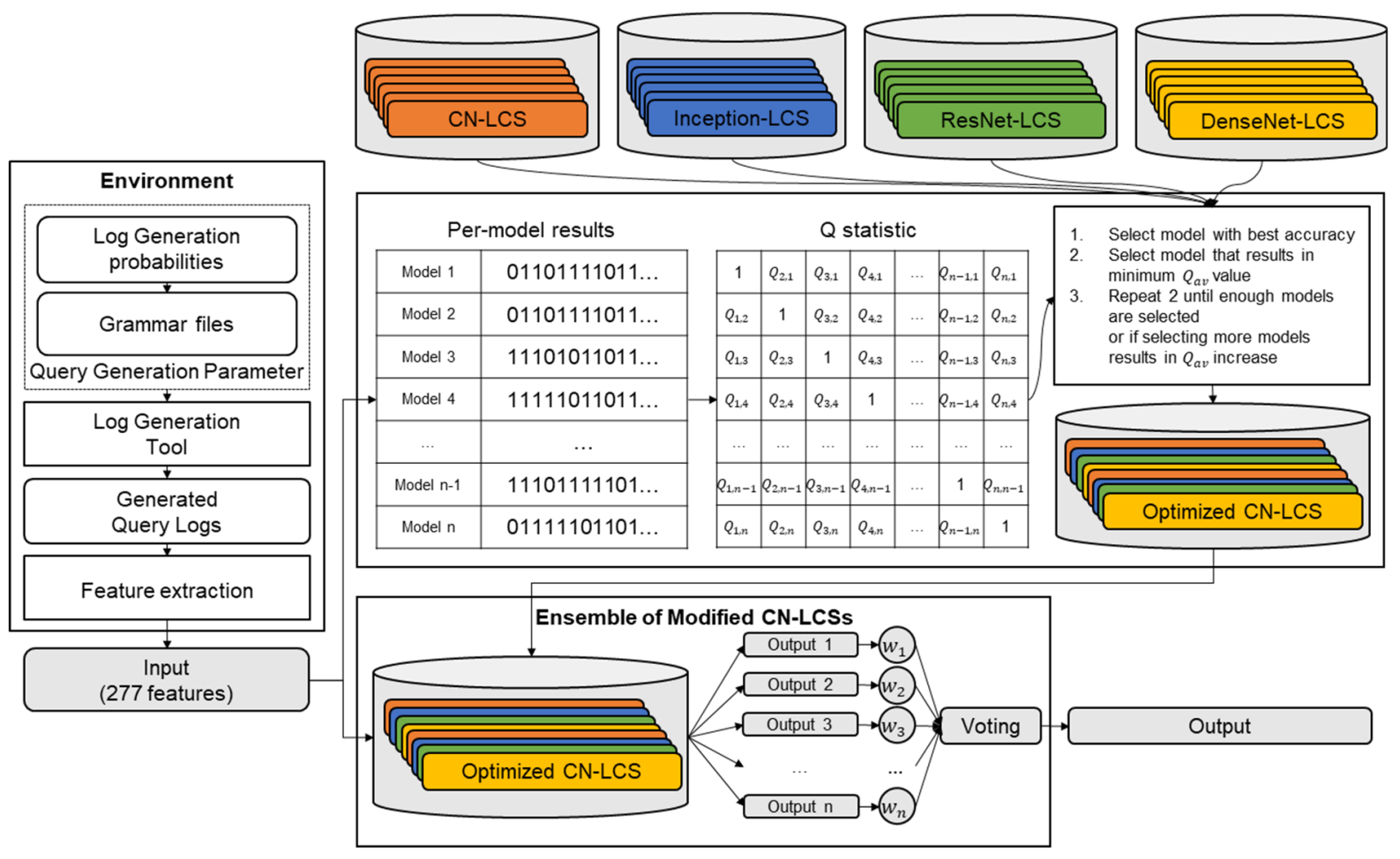

Figure 6. Diagram of the model ensemble component.

\section{Experimental Results}

In this section, we present how the ensemble models the SQL transactions and evaluate the performance with 10-fold cross-validation in terms of classification accuracy, which is followed by quantitative comparison with the relevant deep learning models, including previous CN-LCS models.

\subsection{Implementation}

Deep learning models were implemented using Pytorch [32], with chromosomes stored using Numpy [33]. Models that are not neural network-based were implemented using the default methods provided by the Scikit-learn package [34]. Hyperparameters for each model's training are in Table 9.

Table 9. Model Training Hyperparameters.

\begin{tabular}{cc}
\hline \multicolumn{2}{c}{ Genetic Algorithm Hyperparameter } \\
\hline Number of Generations & 50 \\
\hline Number of Population & 10 \\
\hline Number of Elites & 2 \\
\hline Crossover Rate & 0.5 \\
\hline Mutation Rate & 0.01 \\
\hline Selection Method & Roulette wheel \\
\hline Crossover Method & One-point crossover \\
\hline \multicolumn{2}{c}{ CNN Training Hyperparameter } \\
\hline Optimizer & Adam \\
\hline Learning Rate & 0.001 \\
\hline Number of Epochs & 500 with early stopping \\
\hline
\end{tabular}


During dataset generation, 1000 queries per role (total of 11,000 queries) are generated and pre-processed. The dataset is split 10 -fold, using $90 \%$ of the dataset for training and the other $10 \%$ for testing for each run.

\subsection{SQL Transaction Modeling Performance}

Figure 7 compares the performance of existing machine learning-based query classification models, including our proposed method (Ensemble-LCS). The proposed method achieves the highest classification accuracy of 0.975 , which is higher than other CN-LCS models that use a single architecture for classification.

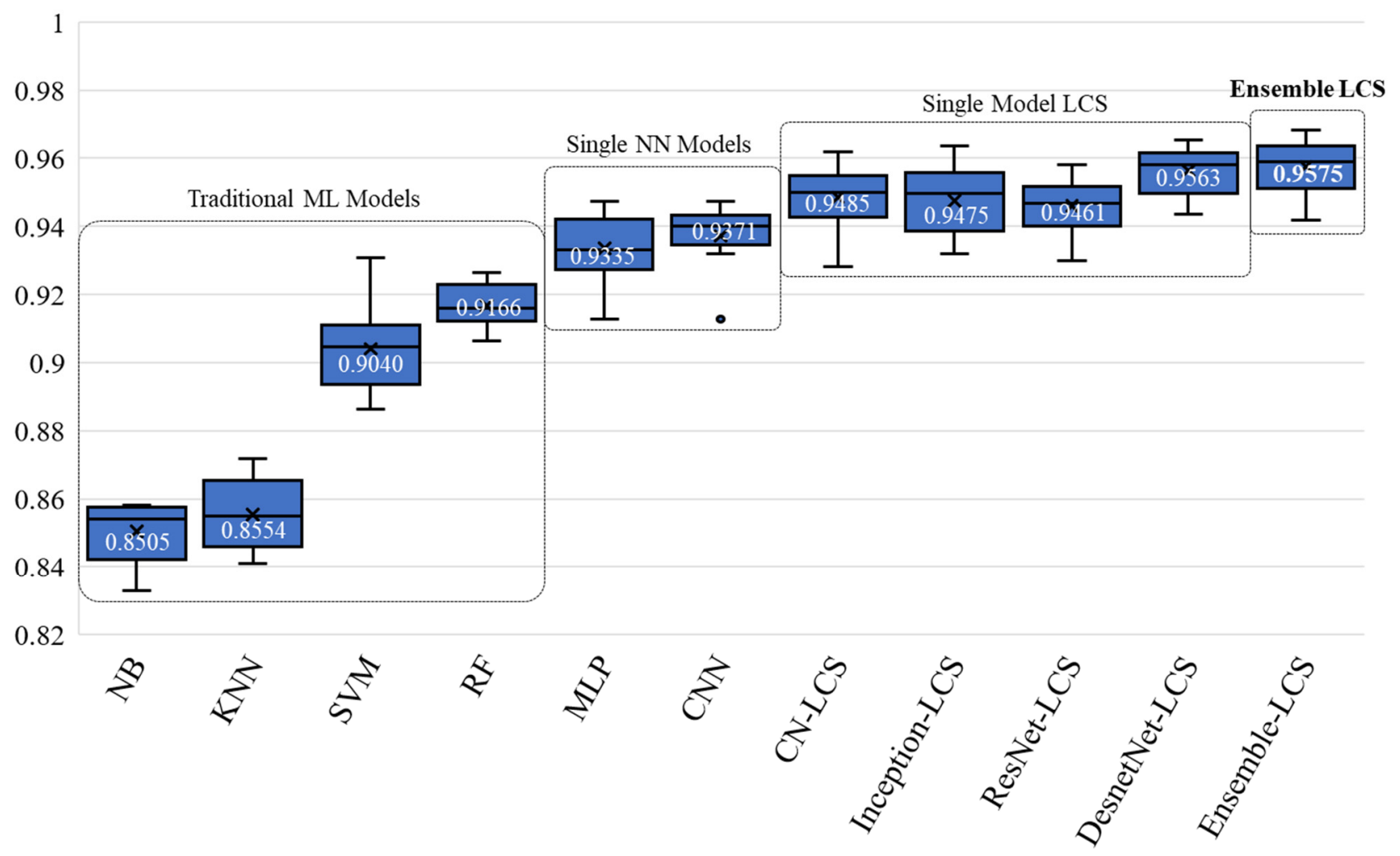

Figure 7. Ten-fold cross-validation with other machine-learning-based classifiers, including the existing combination of GA and deep learning.

Tables 10-14 are the confusion matrix of each LCS model. Darker color indicates how many samples corresponds to the (True, Predicted) pair. While all models show relatively high accuracy, single architecture LCS models showed error instances roles 2-10, with a tendency to classify Trade-status (4) roles as Trade-update (7), Trade-order (6) as Trade-result (10), Trade-update (7) as Trade-lookup (5), and Trade-result (10) as Trade-order (6). The ensembleLCS has reduced overall misclassification. It still misclassifies in cases where all single architecture LCS misclassified, but the reduction in error for roles that single-model LCS struggled, such as role 10, the ensemble-LCS was able to compensate, thus improving overall accuracy.

In several instances, the query was correctly classified by only one of the CN-LCS architectures. Each CN-LCS architecture has an affinity to a certain TPC-E role category and thus had a better chance of correctly classifying queries that were related to that category. The ensemble-LCS was capable of correctly classifying instances, as shown in Table 15. This shows that the ensemble can utilize the varying feature classification characteristic of each of the architectures to reach a more accurate result. 
Table 10. CN-LCS Confusion Matrix.

\begin{tabular}{ccccccccccccc}
\hline True & Predicted & $\mathbf{0}$ & $\mathbf{1}$ & $\mathbf{2}$ & $\mathbf{3}$ & $\mathbf{4}$ & $\mathbf{5}$ & $\mathbf{6}$ & $\mathbf{7}$ & $\mathbf{8}$ & $\mathbf{9}$ & $\mathbf{1 0}$ \\
\hline $\mathbf{0}$ & 1000 & 0 & 0 & 0 & 0 & 0 & 0 & 0 & 0 & 0 \\
\hline $\mathbf{1}$ & 0 & 1000 & 0 & 0 & 0 & 0 & 0 & 0 & 0 & 0 \\
\hline $\mathbf{2}$ & 0 & 0 & 981 & 0 & 0 & 0 & 5 & 7 & 7 & 0 & 0 \\
\hline $\mathbf{3}$ & 0 & 0 & 6 & 990 & 2 & 0 & 0 & 1 & 1 & 0 & 0 \\
\hline $\mathbf{4}$ & 0 & 1 & 0 & 1 & 937 & 17 & 0 & 43 & 0 & 0 & 1 \\
\hline $\mathbf{5}$ & 0 & 5 & 0 & 0 & 1 & 985 & 0 & 0 & 0 & 9 & 0 \\
\hline $\mathbf{6}$ & 0 & 0 & 0 & 0 & 2 & 0 & 840 & 6 & 0 & 0 & 152 \\
\hline $\mathbf{7}$ & 0 & 0 & 0 & 0 & 61 & 126 & 0 & 809 & 0 & 4 & 0 \\
\hline $\mathbf{8}$ & 0 & 0 & 0 & 0 & 0 & 0 & 0 & 11 & 989 & 0 & 0 \\
\hline $\mathbf{9}$ & 0 & 1 & 0 & 0 & 7 & 25 & 1 & 9 & 0 & 957 & 0 \\
\hline $\mathbf{1 0}$ & 0 & 0 & 0 & 0 & 7 & 0 & 28 & 6 & 2 & 11 & 946 \\
\hline
\end{tabular}

Table 11. Inception-LCS Confusion Matrix.

\begin{tabular}{ccccccccccccc}
\hline True & Predicted & $\mathbf{0}$ & $\mathbf{1}$ & $\mathbf{2}$ & $\mathbf{3}$ & $\mathbf{4}$ & $\mathbf{5}$ & $\mathbf{6}$ & $\mathbf{7}$ & $\mathbf{8}$ & $\mathbf{9}$ & $\mathbf{1 0}$ \\
\hline $\mathbf{0}$ & 1000 & 0 & 0 & 0 & 0 & 0 & 0 & 0 & 0 & 0 \\
\hline $\mathbf{1}$ & 0 & 998 & 2 & 0 & 0 & 0 & 0 & 0 & 0 & 0 \\
\hline $\mathbf{2}$ & 0 & 0 & 997 & 0 & 0 & 0 & 0 & 2 & 1 & 0 & 0 \\
\hline $\mathbf{3}$ & 0 & 0 & 8 & 989 & 0 & 0 & 0 & 2 & 1 & 0 & 0 \\
\hline $\mathbf{4}$ & 0 & 1 & 11 & 0 & 929 & 14 & 0 & 45 & 0 & 0 & 0 \\
\hline $\mathbf{5}$ & 0 & 5 & 0 & 0 & 3 & 991 & 0 & 1 & 0 & 0 & 0 \\
\hline $\mathbf{6}$ & 0 & 1 & 10 & 0 & 4 & 1 & 907 & 2 & 0 & 0 & 75 \\
\hline $\mathbf{7}$ & 0 & 0 & 15 & 0 & 19 & 106 & 0 & 858 & 0 & 2 & 0 \\
\hline $\mathbf{8}$ & 0 & 0 & 8 & 0 & 1 & 0 & 0 & 11 & 972 & 0 & 8 \\
\hline $\mathbf{9}$ & 0 & 0 & 0 & 0 & 0 & 34 & 0 & 18 & 0 & 948 & 0 \\
\hline $\mathbf{1 0}$ & 0 & 0 & 4 & 0 & 7 & 5 & 124 & 15 & 1 & 11 & 833 \\
\hline
\end{tabular}

Table 12. ResNet-LCS Confusion Matrix.

\begin{tabular}{|c|c|c|c|c|c|c|c|c|c|c|c|}
\hline True & Predicted & 1 & 2 & 3 & 4 & 5 & 6 & 7 & 8 & 9 & 10 \\
\hline 0 & 1000 & 0 & 0 & 0 & 0 & 0 & 0 & 0 & 0 & 0 & 0 \\
\hline 1 & 0 & 1000 & 0 & 0 & 0 & 0 & 0 & 0 & 0 & 0 & 0 \\
\hline 2 & 0 & 0 & 980 & 1 & 0 & 1 & 0 & 12 & 1 & 5 & 0 \\
\hline 3 & 0 & 0 & 5 & 993 & 1 & 0 & 0 & 0 & 1 & 0 & 0 \\
\hline 4 & 0 & 1 & 0 & 1 & 923 & 14 & 0 & 60 & 0 & 1 & 0 \\
\hline 5 & 0 & 5 & 0 & 0 & 2 & 960 & 0 & 16 & 0 & 17 & 0 \\
\hline 6 & 0 & 0 & 2 & 0 & 1 & 0 & 913 & 0 & 0 & 1 & 83 \\
\hline 7 & 0 & 0 & 2 & 0 & 16 & 92 & 0 & 879 & 0 & 11 & 0 \\
\hline 8 & 0 & 0 & 3 & 0 & 0 & 0 & 2 & 11 & 984 & 0 & 0 \\
\hline 9 & 0 & 0 & 1 & 0 & 0 & 15 & 0 & 7 & 1 & 974 & 2 \\
\hline 10 & 0 & 0 & 0 & 0 & 3 & 3 & 131 & 6 & 0 & 56 & 801 \\
\hline
\end{tabular}


Table 13. DenseNet-LCS Confusion Matrix.

\begin{tabular}{ccccccccccccc}
\hline True & Predicted & $\mathbf{0}$ & $\mathbf{1}$ & $\mathbf{2}$ & $\mathbf{3}$ & $\mathbf{4}$ & $\mathbf{5}$ & $\mathbf{6}$ & $\mathbf{7}$ & $\mathbf{8}$ & $\mathbf{9}$ & $\mathbf{1 0}$ \\
\hline $\mathbf{0}$ & 1000 & 0 & 0 & 0 & 0 & 0 & 0 & 0 & 0 & 0 \\
\hline $\mathbf{1}$ & 0 & 1000 & 0 & 0 & 0 & 0 & 0 & 0 & 0 & 0 \\
\hline $\mathbf{2}$ & 0 & 0 & 992 & 1 & 0 & 0 & 0 & 7 & 0 & 0 & 0 \\
\hline $\mathbf{3}$ & 0 & 0 & 1 & 995 & 2 & 0 & 0 & 1 & 1 & 0 & 0 \\
\hline $\mathbf{4}$ & 0 & 1 & 0 & 0 & 893 & 7 & 0 & 95 & 0 & 4 & 0 \\
\hline $\mathbf{5}$ & 0 & 5 & 0 & 0 & 0 & 951 & 0 & 20 & 0 & 24 & 0 \\
\hline $\mathbf{6}$ & 0 & 0 & 0 & 0 & 2 & 0 & 890 & 2 & 0 & 0 & 106 \\
\hline $\mathbf{7}$ & 0 & 0 & 0 & 0 & 1 & 83 & 0 & 902 & 0 & 14 & 0 \\
\hline $\mathbf{8}$ & 0 & 0 & 0 & 0 & 0 & 0 & 0 & 11 & 989 & 0 & 0 \\
\hline $\mathbf{9}$ & 0 & 1 & 0 & 0 & 0 & 3 & 0 & 1 & 0 & 995 & 0 \\
\hline $\mathbf{1 0}$ & 0 & 0 & 0 & 0 & 4 & 0 & 52 & 9 & 0 & 23 & 912 \\
\hline
\end{tabular}

Table 14. Ensemble-LCS Confusion Matrix.

\begin{tabular}{ccccccccccccc}
\hline True & Predicted & $\mathbf{0}$ & $\mathbf{1}$ & $\mathbf{2}$ & $\mathbf{3}$ & $\mathbf{4}$ & $\mathbf{5}$ & $\mathbf{6}$ & $\mathbf{7}$ & $\mathbf{8}$ & $\mathbf{9}$ & $\mathbf{1 0}$ \\
\hline $\mathbf{0}$ & 1000 & 0 & 0 & 0 & 0 & 0 & 0 & 0 & 0 & 0 \\
\hline $\mathbf{1}$ & 0 & 1000 & 0 & 0 & 0 & 0 & 0 & 0 & 0 & 0 \\
\hline $\mathbf{2}$ & 0 & 0 & 992 & 1 & 0 & 0 & 0 & 5 & 2 & 0 & 0 \\
\hline $\mathbf{3}$ & 0 & 0 & 3 & 996 & 0 & 0 & 0 & 0 & 1 & 0 & 0 \\
\hline $\mathbf{4}$ & 0 & 1 & 0 & 2 & 912 & 10 & 0 & 72 & 0 & 3 & 0 \\
\hline $\mathbf{5}$ & 0 & 5 & 0 & 0 & 0 & 962 & 0 & 13 & 0 & 20 & 0 \\
\hline $\mathbf{6}$ & 0 & 0 & 0 & 0 & 1 & 0 & 880 & 1 & 0 & 0 & 118 \\
\hline $\mathbf{7}$ & 0 & 0 & 1 & 0 & 7 & 94 & 0 & 883 & 0 & 15 & 0 \\
\hline $\mathbf{8}$ & 0 & 0 & 0 & 0 & 0 & 0 & 0 & 11 & 989 & 0 & 0 \\
\hline $\mathbf{9}$ & 0 & 1 & 0 & 0 & 0 & 8 & 0 & 0 & 0 & 991 & 0 \\
\hline
\end{tabular}

A one-way chi-square test with 10 degrees of freedom (number of roles $=1$ ) is performed between the ensemble-LCS and single-model LCS to determine whether the ensemble-LCS shows a significant difference in distribution. The results are shown in Table 16. The ensemble-LCS shows a significant difference, meaning the increase in performance compared to single-architecture models is significant. Thus, we can conclude that the ensemble-LCS shows a significant improvement over previous LCS models.

Table 15. Query samples correctly classified by Model-LCS trained on incomplete query inputs.

\begin{tabular}{|c|c|c|c|}
\hline Prior Correctly Classified Model & Relevant Features of Query & Associated TPC-E Role Category & $\begin{array}{l}\text { Correctly Classified by } \\
\text { Ensemble }\end{array}$ \\
\hline $\mathrm{CNN}$ & $\begin{array}{l}\text { projectionNum.holding_summary; } \\
\text { projectionId.holding_summary; } \\
\text { projectionNum.trade; } \\
\text { projectionId.trade; } \\
\text { whereClauseNum; andOrNum; } \\
\text { stringValuesLength; } \\
\text { stringValueNum; } \\
\text { numericValueNum }\end{array}$ & Customer & Yes \\
\hline
\end{tabular}


Table 15. Cont.

\begin{tabular}{|c|c|c|c|}
\hline Prior Correctly Classified Model & Relevant Features of Query & Associated TPC-E Role Category & $\begin{array}{c}\text { Correctly Classified by } \\
\text { Ensemble }\end{array}$ \\
\hline $\mathrm{CNN}$ & $\begin{array}{l}\text { projectionNum.cash_transaction; } \\
\text { projectionId.cash_transaction; } \\
\text { projectionNum.settlement; } \\
\text { projectionId.settlement; } \\
\text { projectionNum.trade_history; } \\
\text { projectionId.trade_history; } \\
\text { whereClauseNum; orderByNum; } \\
\text { orderByNum.cash_transaction; } \\
\text { orderById.cash_transaction; } \\
\text { andOrNum; stringValuesLength; } \\
\text { stringValueNum; } \\
\text { numericValueNum }\end{array}$ & Customer & Yes \\
\hline Inception & $\begin{array}{l}\text { projectionNum.security; } \\
\text { projectionId.security; } \\
\text { projectionNum.trade; } \\
\text { projectionId.trade; } \\
\text { projectionNum.trade_type; } \\
\text { projectionId.trade_type; } \\
\text { whereClauseNum; orderByNum; } \\
\text { andOrNum; stringValuesLength; } \\
\text { stringValueNum; } \\
\text { numericValueNum }\end{array}$ & Trade & Yes \\
\hline Inception & $\begin{array}{l}\text { projectionNum.trade; } \\
\text { projectionId.trade; } \\
\text { whereClauseNum; orderByNum; } \\
\text { andOrNum; stringValuesLength; } \\
\text { stringValueNum; } \\
\text { numericValueNum }\end{array}$ & Trade & Yes \\
\hline ResNet & $\begin{array}{l}\text { projectionNum.last_trade; } \\
\text { projectionId.last_trade; } \\
\text { stringValuesLength; } \\
\text { stringValueNum }\end{array}$ & Market & Yes \\
\hline ResNet & $\begin{array}{l}\text { projectionNum.last_trade; } \\
\text { projectionId.last_trade; } \\
\text { projectionNum.security; } \\
\text { projectionId.security; } \\
\text { projectionNum.trade_history; } \\
\text { projectionId.trade_history; } \\
\text { whereClauseNum; orderByNum; } \\
\text { andOrNum; stringValuesLength; } \\
\text { stringValueNum; } \\
\text { numericValueNum }\end{array}$ & Market & Yes \\
\hline
\end{tabular}

Table 16. The chi-square values CN-LCS ensemble and single-model CN-LCS.

\begin{tabular}{ccccc}
\hline & Comparative & $\begin{array}{c}\text { Chi-Square } \\
\text { Statistic }\end{array}$ & $p$-Value & Significance \\
\hline \multirow{2}{*}{ Ensemble-LCS } & CN-LCS & 51.3146 & $1.5278 \times 10^{-7}$ & $p<0.01$ \\
\cline { 2 - 5 } & Inception-LCS & 19.1691 & 0.0382 & $p<0.05$ \\
\cline { 2 - 5 } & ResNet-LCS & 16.7888 & 0.0792 & $p<0.1$ \\
\cline { 2 - 5 } & DenseNet-LCS & 23.8290 & 0.0081 & $p<0.01$ \\
\hline
\end{tabular}

\subsection{Model Adaptability Performance}

To measure the adaptability of the Ensemble-LCS model, we create an imbalanced dataset. Queries with Role 10 (Trade-Result) are divided based on the queryMode feature. Approximately 50\% of all Role 10 queries have a queryMode value of 1, followed by 2 to 4. The models are trained on a dataset where Role 10 inputs with queryMode value 1 is removed, simulating incomplete or imbalanced input data. Then, the models are evaluated with a dataset with all Role 10 inputs included. 
Table 17 shows the result of the experiment. The ensemble-LCS shows higher accuracy for Role 10 queries compared to other models. While the accuracy for other roles was lower than for the CN-LCS model, it still showed relatively higher accuracy than other models. Showing that the ensemble-LCS model has better robustness against data imbalance.

Table 17. Accuracy of each Model-LCS trained on incomplete query inputs.

\begin{tabular}{ccc}
\hline Model & Role 10 Accuracy & Other Role Accuracy \\
\hline CN-LCS & 0.5000 & 0.8889 \\
\hline Inception-LCS & 0.5020 & 0.8465 \\
\hline ResNet-LCS & 0.3050 & 0.7363 \\
\hline DenseNet-LCS & 0.5260 & 0.8734 \\
\hline Ensemble-LCS & 0.6640 & 0.8874 \\
\hline
\end{tabular}

\section{Concluding Remarks}

In this paper, we proposed an ensemble-LCS model for database IDS using RBACbased query classification. This method combines multiple heterogeneous model architectures into an ensemble to exploit each model's characteristics while supplementing each model's weakness. The proposed method showed improved accuracy compared to single-model-based LCS methods; we verified the statistical significance of the proposed method using 10-fold cross-validation and chi-square validation. The proposed method also displayed adaptability when the provided data was imbalanced. This shows that diversity-guided ensemble-LCS can be used to develop improved IDS using RBAC-based query role classification.

Future work will include introducing additional CNN models to be included in the ensemble to improve overall diversity and increase classification accuracy. Additionally, the proposed method will have to be tested on other datasets to validate whether the proposed method can be generalized to other database schemas.

Author Contributions: S.-J.B.; H.-B.K. and S.-B.C. have participated in preparing the research from the beginning to the end, such as establishing research design, methods, and analysis. S.-J.B.; H.-B.K. and S.-B.C. discussed and finalized the analysis results to prepare the manuscript according to the progress of research. All authors contributed equally to this work. All authors have read and agreed to the published version of the manuscript.

Funding: This work was supported by an IITP grant funded by the Korean government (MSIT) (No. 2020-0-01361, Artificial Intelligence Graduate School Program (Yonsei University)) and Air Force Defense Research Sciences Program funded by Air Force Office of Scientific Research.

Conflicts of Interest: The authors declare no conflict of interest.

\section{References}

1. Khraisat, A.; Gondal, I.; Vamplew, P.; Kamruzzaman, J. Survey of intrusion detection systems: Techniques, datasets and challenges. Cybersecurity 2019, 2, 20. [CrossRef]

2. Sagar, R.; Jhaveri, R.; Borrego, C. Applications in Security and Evasions in Machine Learning: A Survey. Electronics 2020, 9, 97. [CrossRef]

3. Rawat, D.B. Journal of Cybersecurity and Privacy: A New Open Access Journal. J. Cybersecur. Priv. 2021, 1, 195-198. [CrossRef]

4. Ronao, C.A.; Cho, S.B. Anomalous query access detection in RBAC-administered databases with random forest and PCA. Inf. Sci. 2016, 369, 238-250. [CrossRef]

5. Bertino, E.; Sandhu, R. Database security-concepts, approaches, and challenges. IEEE Trans. Dependable Secur. Comput. 2005, 2, 2-19. [CrossRef]

6. Safa, N.S.; Maple, C.; Watson, T.; Von Solms, R. Motivation and opportunity based model to reduce information security insider threats in organisations. J. Inf. Secur. Appl. 2018, 40, 247-257. [CrossRef]

7. Bu, S.-J.; Cho, S.-B. A convolutional neural-based learning classifier system for detecting database intrusion via insider attack. Inf. Sci. 2020, 512, 123-136. [CrossRef]

8. Saxena, N.; Hayes, E.; Bertino, E.; Ojo, P.; Choo, K.-K.R.; Burnap, P. Impact and Key Challenges of Insider Threats on Organizations and Critical Businesses. Electronics 2020, 9, 1460. [CrossRef] 
9. Pan, Z.; Hariri, S.; Pacheco, J. Context aware intrusion detection for building automation systems. Comput. Secur. 2019, 85, 181-201. [CrossRef]

10. Buehrer, G.; Weide, B.W.; Sivilotti, P.A. Using parse tree validation to prevent SQL injection attacks. In Proceedings of the 5th International Workshop on Software Engineering and Middleware, Lisbon, Portugal, 5-6 September 2005; pp. $106-113$.

11. Ahmim, A.; Maglaras, L.; Ferrag, M.A.; Derdour, M.; Janicke, H. A novel hierarchical intrusion detection system based on decision tree and rules-based models. In Proceedings of the 2019 15th International Conference on Distributed Computing in Sensor Systems (DCOSS), Santorini Island, Greece, 29-31 May 2019; pp. 228-233.

12. Xue, B.; Zhang, M.; Browne, W.N.; Yao, X. A survey on evolutionary computation approaches to feature selection. IEEE Trans. Evol. Comput. 2015, 20, 606-626. [CrossRef]

13. Kim, M.Y.; Lee, D.H. Data-mining based SQL injection attack detection using internal query trees. Expert Syst Appl. 2014, 41, 5416-5430. [CrossRef]

14. Dam, H.H.; Abbass, H.A.; Lokan, C.; Yao, X. Neural-based learning classifier systems. IEEE T Knowl. Data En. 2008, 20, 26-39. [CrossRef]

15. Kim, T.-Y.; Cho, S.-B. CNN-LSTM Neural Networks for Anomalous Database Intrusion Detection in RBAC-Administered Model. In International Conference on Neural Information Processing; Springer: Cham, Germany, 2019; pp. 131-139. [CrossRef]

16. Khare, N.; Devan, P.; Chowdhary, C.L.; Bhattacharya, S.; Singh, G.; Singh, S.; Yoon, B. SMO-DNN: Spider Monkey Optimization and Deep Neural Network Hybrid Classifier Model for Intrusion Detection. Electronics 2020, 9, 692. [CrossRef]

17. Selvakumar, B.; Muneeswaran, K. Firefly algorithm based feature selection for network intrusion detection. Comput. Secur. 2019, 81, 148-155. [CrossRef]

18. Choi, S.-G.; Cho, S.-B. Evolutionary Reinforcement Learning for Adaptively Detecting Database Intrusions. Log. J. Igpl. 2020, 28, 449-460. [CrossRef]

19. Bu, S.-J.; Cho, S.-B. A Hybrid System of Deep Learning and Learning Classifier System for Database Intrusion Detection. In Hybrid Artificial Intelligent Systems; Springer: Cham, Switzerland, 2017; pp. 615-625.

20. Kim, T.-Y.; Cho, S.-B. Particle Swarm Optimization-Based CNN-LSTM Networks for Anomalous Query Access Control in RBAC-Administered Model. In Hybrid Artificial Intelligent Systems; Springer: Cham, Switzerland, 2019; pp. $123-132$.

21. Bu, S.-J.; Cho, S.-B. Genetic Algorithm-Based Deep Learning Ensemble for Detecting Database Intrusion via Insider Attack. In Hybrid Artificial Intelligent Systems; Springer: Cham, Switzerland, 2019; pp. 145-156.

22. Kim, J.Y.; Cho, S.B. Exploiting deep convolutional neural networks for a neural-based learning classifier system. Neurocomputing 2019, 354, 61-70. [CrossRef]

23. Szegedy, C.; Liu, W.; Jia, Y.; Sermanet, P.; Reed, S.; Anguelov, D.; Erhan, D.; Vanhoucke, V.; Rabinovich, A. Going deeper with convolutions. In Proceedings of the IEEE Conference on Computer Vision and Pattern Recognition, Boston, MA, USA, 8-10 June 2015; pp. 1-9.

24. He, K.; Zhang, X.; Ren, S.; Sun, J. Deep residual learning for image recognition. In Proceedings of the IEEE Conference on Computer Vision and Pattern Recognition, Las Vegas, NV, USA, 27-30 June 2016; pp. 770-778.

25. Khraisat, A.; Gondal, I.; Vamplew, P.; Kamruzzaman, J.; Alazab, A. Hybrid intrusion detection system based on the stacking ensemble of c5 decision tree classifier and one class support vector machine. Electronics 2020, 9, 173. [CrossRef]

26. Khraisat, A.; Gondal, I.; Vamplew, P.; Kamruzzaman, J.; Alazab, A. A Novel Ensemble of Hybrid Intrusion Detection System for Detecting Internet of Things Attacks. Electronics 2019, 8, 1210. [CrossRef]

27. Chen, S.; Ailamaki, A.; Athanassoulis, M.; Gibbons, P.B.; Johnson, R.; Pandis, I.; Stoica, R. TPC-E vs. TPC-C: Characterizing the new TPC-E benchmark via an I/O comparison study. ACM Sigmod. Record 2011, 39, 5-10. [CrossRef]

28. Huang, G.; Liu, Z.; Van Der Maaten, L.; Weinberger, K.Q. Densely connected convolutional networks. In Proceedings of the IEEE Conference on Computer Vision and Pattern Recognition, Honolulu, HI, USA, 21-24 July 2017; pp. 4700-4708.

29. Díez-Pastor, J.F.; Rodríguez, J.J.; García-Osorio, C.I.; Kuncheva, L.I. Diversity techniques improve the performance of the best imbalance learning ensembles. Inf. Sci. 2015, 325, 98-117. [CrossRef]

30. Kuncheva, L.I.; Whitaker, C.J. Measures of Diversity in Classifier Ensembles and Their Relationship with the Ensemble Accuracy. Mach. Learn. 2003, 51, 181-207. [CrossRef]

31. Yule, G.U. VII. On the association of attributes in statistics: With illustrations from the material of the childhood society, \&c. Philos. Trans. R. Soc. London. Ser. A Contain. Pap. A Math. Or Phys. Character 1900, 194, 257-319.

32. Paszke, A.; Gross, S.; Massa, F.; Lerer, A.; Bradbury, J.; Chanan, G.; Killeen, T.; Lin, Z.; Gimelshein, N.; Antiga, L. Pytorch: An imperative style, high-performance deep learning library. Adv. Neural Inf. Processing Syst. 2019, 32, 8026-8037.

33. Harris, C.R.; Millman, K.J.; van der Walt, S.J.; Gommers, R.; Virtanen, P.; Cournapeau, D.; Wieser, E.; Taylor, J.; Berg, S.; Smith, N.J. Array programming with NumPy. Nature 2020, 585, 357-362. [CrossRef] [PubMed]

34. Pedregosa, F.; Varoquaux, G.; Gramfort, A.; Michel, V.; Thirion, B.; Grisel, O.; Blondel, M.; Prettenhofer, P.; Weiss, R.; Dubourg, V. Scikit-learn: Machine learning in Python. J. Mach. Learn. Res. 2011, 12, 2825-2830. 